\title{
Ultrastructural, Histochemical, Cytological Study of Retina of Aborted Fetus of Various Weeks of Gestation - an Anatomical Perspective with Implications on Patients with Retinitis Pigmentosa
}

Arpan HALDAR ${ }^{\mathrm{a}}$, Kaushik SADHUKHAN ${ }^{\mathrm{b}}$, Subhrajyoti NASKAR ${ }^{\mathrm{c}}$

aDepartment of Anatomy, AIIMS DEOGHAR, India

bDepartment of Ophthalmology, AIIMS PATNA, India

'Department of Community Medicine, Diamond Harbour Government Medical College, West Bengal, India

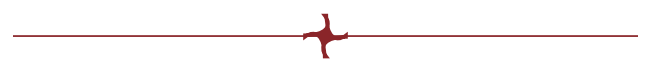

\begin{abstract}
Aim: To study the ultrastructural, histochemical, cytological features of retina in aborted fetuses of different gestational age and its probable implication in the disease process of retinitis pigmentosa.

Methodology: This is a prospective randomized cross sectional study that has been carried out in AIIMS Bhubaneswar from June 2017 to May 2019, jointly by the Department of Anatomy and Obstetrics and Gynaecology, AIIMS, Bhubaneswar, India, after proper institutional approval. Three fetuses from each trimester were taken into the present study; their retina was collected and subsequently sent for cytological, immunohistochemical and ultrastructural explorations. Detailed information from all explorations were collected and properly documented.

Results: Fetuses whose retinas that have been shown to contain very few to no rod cells and short-sized cone cells might tend to develop retinitis pigmentosa after birth. Moreover, those cone cells have been shown to contain melanolysosome, phagosomes, autophagic vacuoles, and membranous whorls.
\end{abstract}

Keywords: rod cells, cone cells, retinitis pigmentosa, retinal pigment epithelium, opsin.

\footnotetext{
Address for correspondence:

Subhrajyoti Naskar, MD, Associate Professor

Department of Community Medicine, Diamond Harbour Government Medical College, West Bengal, India

Postal address: Sahebpara near power house qtr.

P.O. and P.S.: Sonarpur, Kolkata 700150, Pin 700150, India

Tel.: 9830145803, email: subhro79@gmail.com
} 


\section{INTRODUCTION}

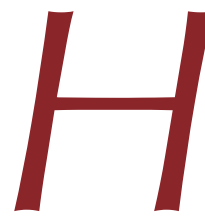

uman retina is formed by very complex but coordinated mechanisms that include cell division, differentiation, migration, intercommunication as well as cell death. Photoreceptor cells along with adjacent retinal pigment epithelium construct one structurally and functionally integrated unit which serves the purpose of vision. A phototransduction molecule comprising opsin (type specific protein) and 11-cis retinal helps in mediating the capture of light. Human cones are responsible for bright light illumination and contains three types of opsin for long- $(\mathrm{L}=564 \mathrm{~nm})$, medium$(\mathrm{M}=533 \mathrm{~nm})$, and short- $(\mathrm{S}=437 \mathrm{~nm})$ wavelength light. On the contrary, rods act in dim light illumination but contain single rod opsin (ROp) that is sensitive to blue-green light $(500 \mathrm{~nm})$ with maximum effect.

A very precise spatially arranged mosaic pattern is characteristic for human rods and cones (1-3). The center of the fovea has only $L$ and $M$ cones, whereas rod cell population in the peripheral retina is 20 times that of cone cells $(2,3)$. Human visual system is very much reliant on that photoreceptor mosaic pattern, although how it generates vision is still beyond our knowledge (4). Foveal depression occurs between 24 weeks of gestation and 45 months of postnatal age due to the migration of maximum number of photoreceptor cells in the inner retinal layer away from the center of the developing fovea; surprisingly, cones migrate in the opposite direction, causing the central foveal cone mosaic to become devoid of rod cells, where cones are crowded, more elongated and densely packed (5-7). In the human eye, the central part of the retina is formed at around 20 weeks of gestation, while peripheral retina develops during a comparatively longer time (up to 40-41 weeks of gestation).

Retinitis pigmentosa (RP), cone-rod dystrophy, leber congenital amaurosis, etc are the result of genetic defects in human rod photoreceptor cells and their manifestation may range from tunnel vision or night blindness to complete blindness.

Retinitis pigmentosa is initially hallmarked by rod cell depletion, which is characterized by night blindness, followed by cone cell death leading to central visual loss.
Autosomal recessive $\mathrm{RP}$, which is the commonest variant, is associated with mutations in ROp and photoreceptor specific tubby like protein (TULP) (8), but the autosomal dominant type results from missense mutations in NRL (neural retinal leucine) zipper genes (9).

\section{METHODOLOGY}

This prospective randomized cross-sectional hospital-based study was jointly conducted by the Department of Anatomy and Obstetrics and Gynecology, AlIMS, Bhubaneswar, India, over the period of two years, from June 2017 to May 2019.

Retinas from three fetuses per each of the three gestation trimesters (a total of nine fetuses) were collected. Retinas from the eyes of aborted human fetuses of the first, second and third trimesters of gestation that did not show any evidence of external morphological abnormality were collected in the Department of Obstetrics and Gynecology, AIIMS, Bhubaneswar, India, after therapeutic abortion, which was used in the present study after receiving both the approval of the Institutional Ethical Committee of Human Research (IEC) and Institute Research Board (IRB) of AIIMS, Bhubaneswar, India, and written informed consent of the legal guardian as per institutional guidelines, all of which being kept in the Fetal Death Register, Department of Anatomy, AIIMS Bhubaneswar, India (Table 1).

For the cytological study, fetuses were dissected by a dissecting microscope and $2 \mathrm{~mm}$ retinal pieces were immediately fixed in $10 \%$ for-

TABLE 1. Fetal specimen characteristics

\begin{tabular}{|l|l|l|l|l|l|}
\hline No. & $\begin{array}{l}\text { Age (weeks } \\
\text { of gestation) }\end{array}$ & $\begin{array}{l}\text { Weight } \\
\text { (grams) }\end{array}$ & $\begin{array}{l}\text { Length } \\
(\mathbf{c m})\end{array}$ & $\begin{array}{l}\text { Surface area } \\
\text { (sq mm) }\end{array}$ & $\begin{array}{l}\text { Percentage of } \\
\text { sampled area }\end{array}$ \\
\hline 1. & 15 & 61 & 13 & 71 & 1.6 \\
\hline 2. & 15 & 73 & 13 & 72 & 1.7 \\
\hline 3. & 16 & 90 & 14 & 88 & 1.4 \\
\hline 4. & 17 & 125 & 16 & 95 & 1.4 \\
\hline 5. & 18 & 147 & 17 & 115 & 1.7 \\
\hline 6. & 20 & 177 & 23 & 212 & 1.8 \\
\hline 7. & 23 & 265 & 33 & 223 & 1.8 \\
\hline 8. & 24 & 270 & 34 & 231 & 1.8 \\
\hline 9. & 25 & 288 & 34 & 229 & 1.8 \\
\hline
\end{tabular}




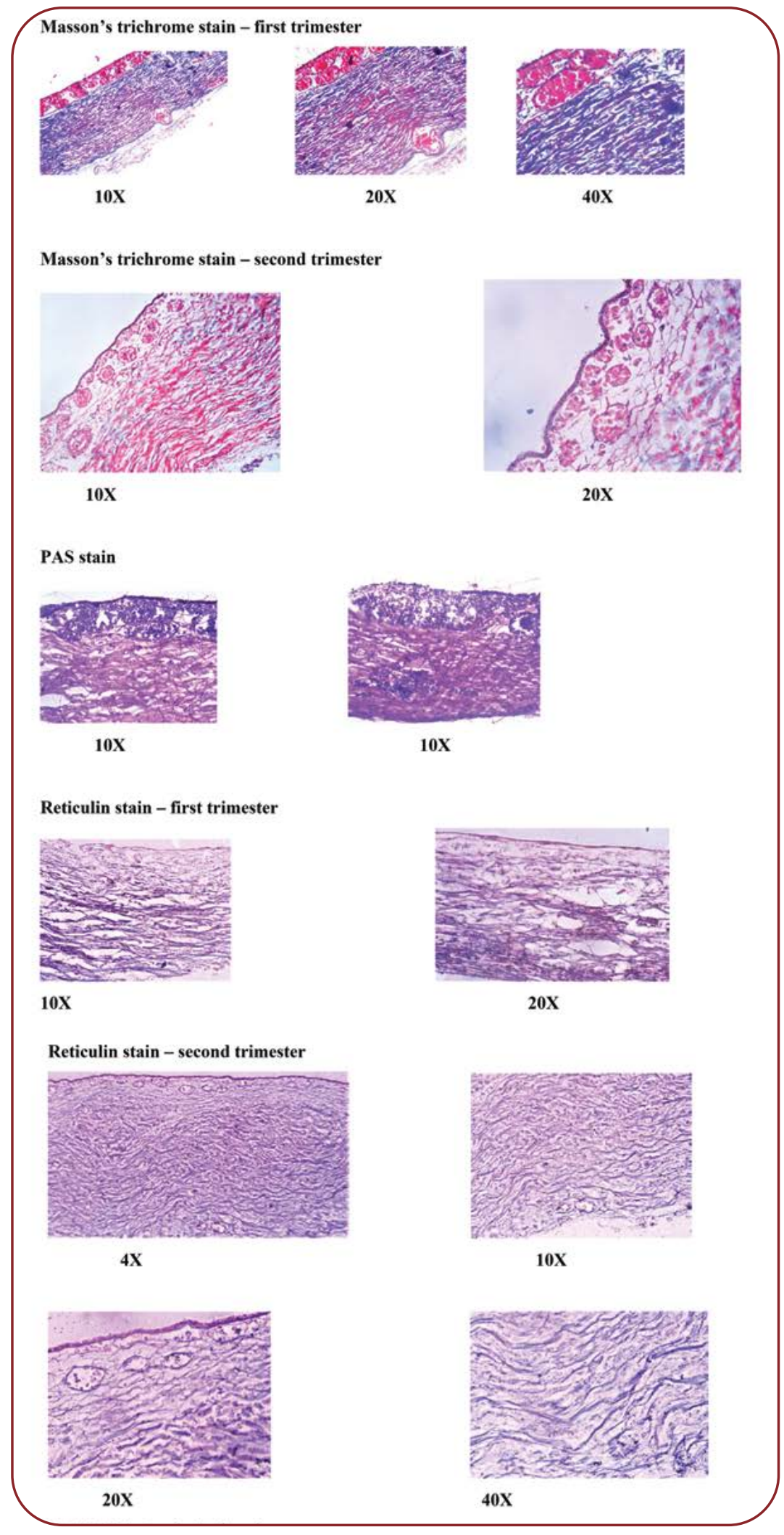

FIGURE 1. Cytological study

malin for 24 hours and subsequently processed for routine histological procedures using special stains such as Masson's trichrome, PAS and re- ticulin stain; afterwards, photomicrographs were taken by camera using a microscope adapter (Figure 1). 


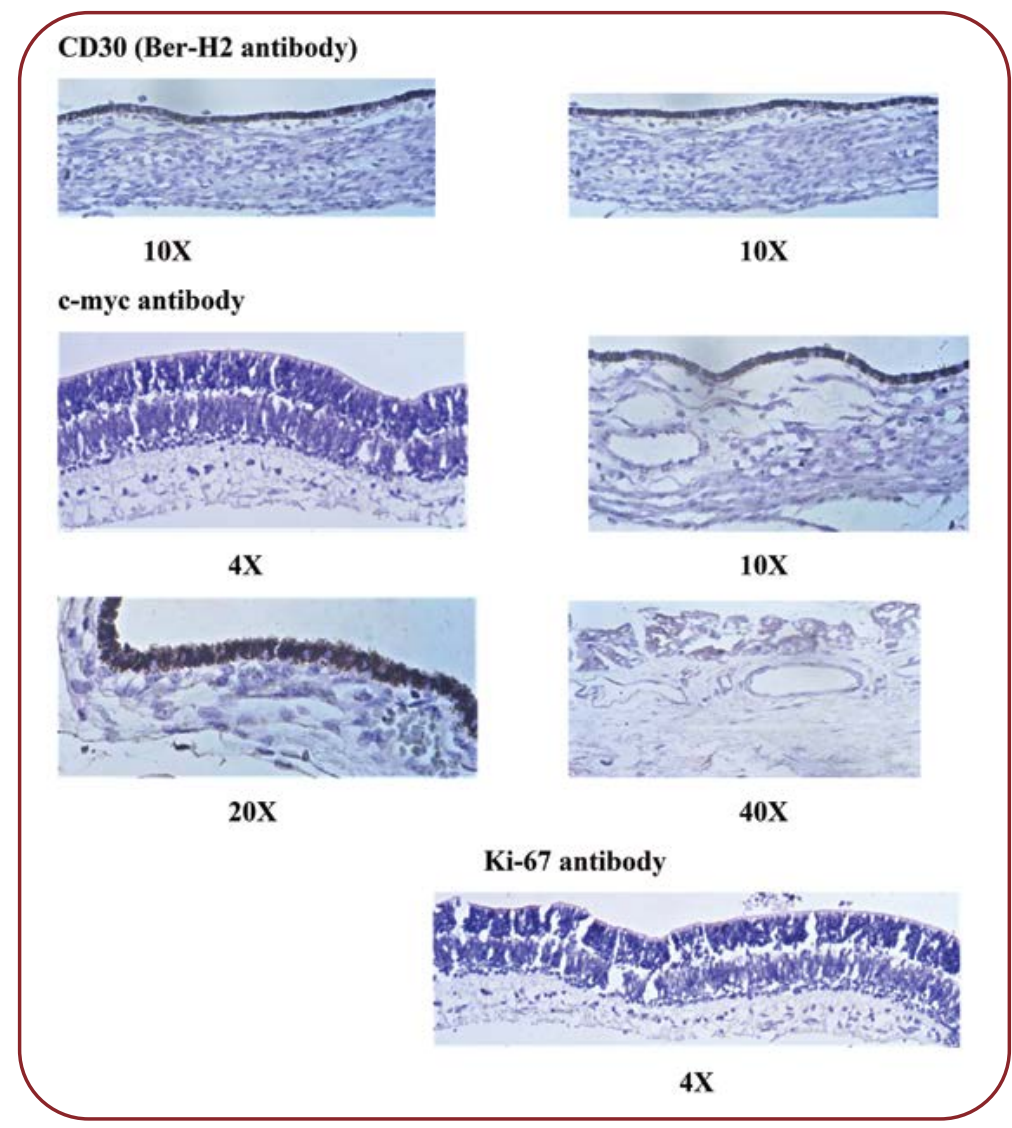

FIGURE 2. Immunohistochemical study

For the immunohistochemical study, $3 \mathrm{~mm}$ sections of retina were fixed in $10 \%$ neutral buffered formalin (NBF) for seven hours and then subjected to routine processing and paraffin embedding. Unstained sections were then heated for antigen retrieval from those tissues which were immersed in DAKO target retrieval solution. Deparaffinized sections were incubated with $3 \%$ hydrogen peroxide for five minutes to block and inhibit the endogenous enzymes and avoid non-specific binding of antigen to the cell surface, followed by sequential 10-min incubations with xylene and alcohol. Subsequently, they were placed in a pressure cooker for 20 minutes (heat-induced epitope retrieval, HIER) to unmask the antigen epitopes and thus allow antibody binding; then, biotinylated link antibody and peroxidase-labelled streptavidin were used to augment the antigen expression on the tissue surface. A modified labelled avidin-biotin immunohistochemical staining with triple antibodies c-myc, Ki-67 and Ber-H2 was performed with immunoperoxidase kit (DAKO) on DAKO auto stainer (Figure 2). Staining was completed after 10-min incubation with DAKO 3,3'-diaminobenzidine (DAB) chromogen, which was used as signal enhancer. All cases were coded and immunostaining grading was performed on a sliding scale of $1+$ to $4+$, according to the percentage of reactive cells: $0=$ no staining; $1+=1-25 \% ; 2+=26-50 \%$; $3+=51-75 \%$; and $4+>76 \%$.

For the ultrastructural study, retinal tissue from human fetus eyes was cut in $2 \times 2 \mathrm{~mm}$ pieces and put in primary fixative (Karnovsky's fixative) $-2.5 \%$ glutaraldehyde $+2 \%$ paraformaldehyde in $0.1 \mathrm{M} \mathrm{PB}\left(\mathrm{pH} \mathrm{7.4)}\right.$ - for 12 hours at $4^{\circ} \mathrm{C}$, followed by secondary fixation in $1 \%$ OsO4 for one hour at $4^{\circ} \mathrm{C}$. The tissue was then dehydrated in ascending grades of alcohol at $4^{\circ} \mathrm{C}$ and kept in acetone at room temperature, followed by processing with xylene. Epoxy resin infiltration was done with descending toluene: ascending resin ratio for better tissue infiltration. The resin block was then cut into 50-70 nm sections by ultramicrotome knife. Double staining was done by aqueous uranyl acetate and alkaline lead citrate. Slides were then viewed under transmission electron microscopy (TEM) and photomicrographs were taken (Figure 3). 


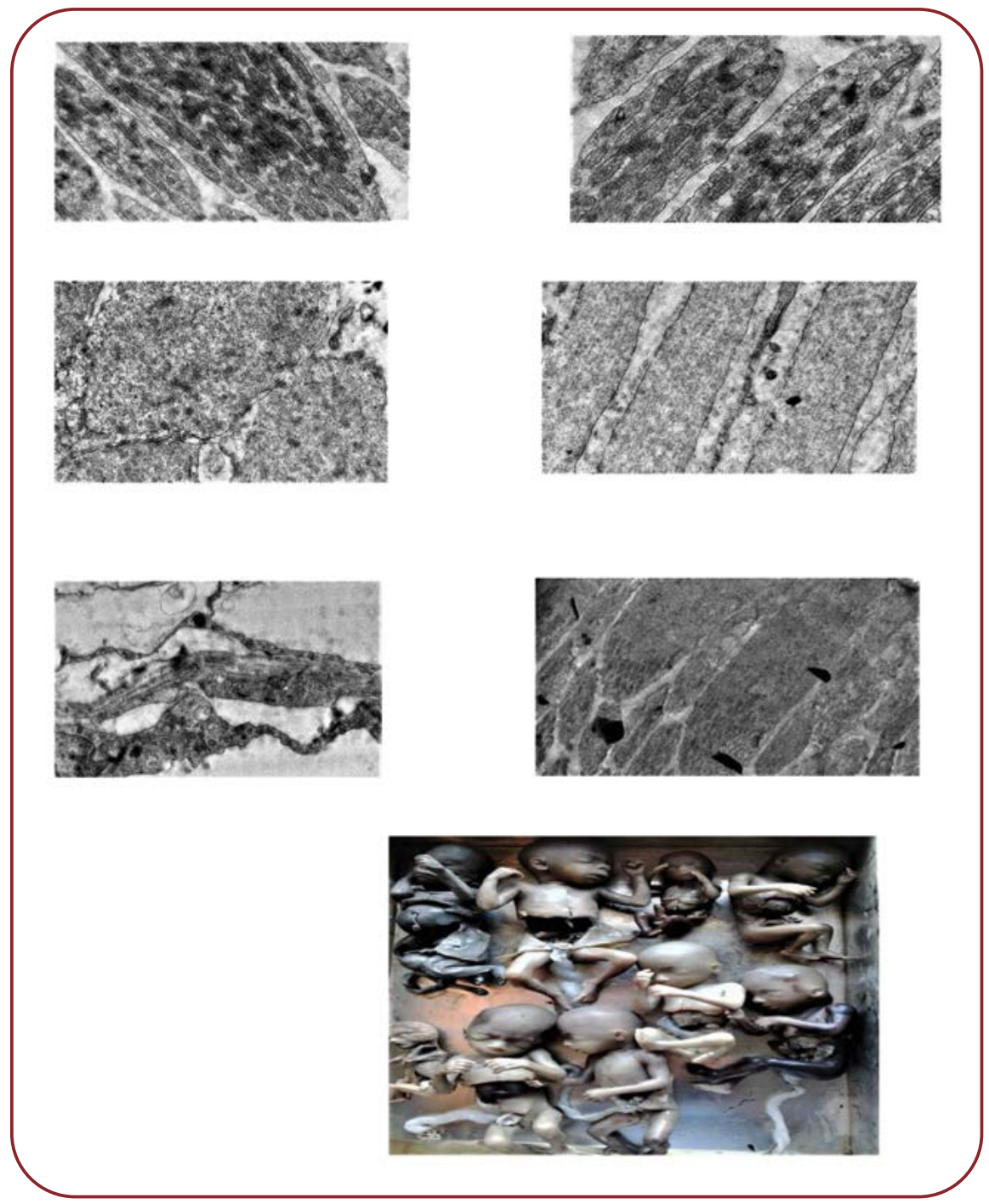

FIGURE 3.

Transmission electron microscopy (TEM) study (photographs collected from nine fetuses)

Immunohistochemical study

A few anaphase mitotic cells are recognised by migrating condensed chromatids and seen adjacent to the intraretinal space which is separating the neural retina from the pigmented retina. Surrounding neuroblasts show oval euchromatic nuclei. Immune positive cells are seen in the pigmented retina and the outer neuroblast zone of the central neural retina, while in the peripheral part of the neural retina they are found throughout the whole thickness of the zone of primitive neuroblast nuclei. In the initial weeks of gestation, pigmented retina is reduced in thickness to a simple columnar epithelium. The neuroblast nuclear layer now consists of the outer neuroblast zone of oval nuclei and inner zone of more spherical nuclei. The nerve fibre layer is located internal to the inner neuroblast nuclei. The central neural retina consists of the outer neuroblast zone, inner neuroblast zone and nerve fibre layer. The peripheral (anterior) part of the neural retina consists only of the zone of primitive neuroblast nuclei, being less mature and thinner than its central (posterior) part. The two retinal layers are separated by an intraretinal space. The outer layer of the optic cup forms the pigmented retina that consists of a pseudostratified columnar pigmented epithelium. The inner layer (future neural retina) comprises the zone of neuroblast nuclei occupying the outer three-fourths of the epithelium and the inner marginal zone which is free of nuclei. The two retinal layers are separated by an intraretinal space. Immune positive cells, characterised by brown nuclear stai- 
ning, are found in the pigmented retina and the neuroblast zone of the neural retina. The most intensive staining is seen in mitotic figures lining the intraretinal space. In the neuroblast zone of the neural retina as well as in the pigmented retina, positive cells show various nuclear staining patterns: agranular pattern with accentuation of the nucleoli; a more distinct, large, dot-like pattern with prominent nucleoli, and the most intensive staining of chromosomes in mitotic figures lining the lumen. Non-proliferating, immune negative cells have oval pale-stained nuclei with prominent nucleoli.

\section{Ultrastructural study}

Extracellular spaces of mesenchyme overlie the posteromedial base of the retinal disc. Basement membrane material partially covers the base of the cellular processes of the retina. The ventricular layer of the embryonic retinal disc has a series of zonulae adherents beyond which cytoplasmic excrescences with cilia protrude into the optic ventricle. The inner end process of a Muller-cell and the basement membrane are tented inwardly where collagen fibrils connect to the primary vitreous. The Muller-cell cytoplasm contains small matrix particles and intermediate filaments. The basement membrane and vitreous body are in the upper left in the inner retina. The nerve fibre layer has fascicles of ganglion-cell axons in its outer half. Muller-cell processes and nuclei are denser than the numerous ganglion-cell axons and nuclei. Inner Muller-cell processes contain rough endoplasmic reticulum and intermediate filaments. Cells by the external limiting membrane (zonulae adherents) are dense and morphologically undifferentiated. A centriole is seen in a cell of the outer neural retina. The pigment epithelium is connected to the neural retina by junctional complex components. Intertwining Muller-cell processes ending on the basement membrane of retina vary in density. The dense cytoplasm on the left may include some intermediate filaments, while the lucent glial process on the right contains many intermediate filaments. The inner Muller-cell processes of the retina contain smooth endoplasmic reticulum, intermediate filaments and glycogen particles. A dense Muller-cell separates immature cone cells in the outer retina. The dense outer Muller-cell processes contain glycogen particles.

\section{Implications in retinitis pigmentosa}

Rod photoreceptors are very few to nil in number. Cone cells, which are located in the fovea and perifovea, have very short outer and inner segments and characteristically contain autophagic vacuoles and many filaments. Retinal pigment epithelium (RPE) cells adjacent to cone cells are larger and contain large number of melanolysosomes along with phagosomes. Lipofuscin granules are occasionally found in pigment epithelium cells. Subretinal spaces often have membranous whorls. But anterior to perifovea RPE cells do not contain any melanolysosomes.

\section{DISCUSSION}

n the present study we mainly focus on how rod photoreceptors develop and how rod-associated proteins express in the context of future development of retinitis pigmentosa.

At about 11 weeks of gestation, rods begin to appear with the expression of NRL, whereas at about 15 weeks of gestation differentiation along with maturation of rod cells are initiated marked by expression of ROp mRNA; TULP is the last protein to be expressed at about 17 weeks of gestation. Gradually, rod cells acquire the adult shape characterized by the long outer segment which is connected to the inner segment by thin cilium.

In the central part of adult retina, antibodies to $L$ and $M$ opsin and ROp markedly label the outer segment (OS). Rod cells are more numerous in the peripheral retina and at the same time they have longer OS compared to cone cells in that particular zone, whereas in the central retina cones are predominant in terms of both number and size. Recoverin labels both the cytoplasm and OS of cone and rod cells, while peripherin stains only the OS of both types of cells, not also the cytoplasm. Interestingly, arrestin labels the cytoplasm of entire rod cells. Interphotoreceptor retinoid binding protein (IRBP) again labels the interphotoreceptor matrix surrounding the outer- and inner-segments up to the outer limiting membrane.

First evidence of fovea development is discovered at about 10.5-11 weeks of gestation, when it has five layers and the outer nuclear layer (ONL) only contains the cone cells, thus forming the pure cone area (PCA); but at about 
midpoint of gestation, ONL have both single layer of large cones as well as single layer of small rod cells. However, photoreceptors acquire more mature morphology as the age of fetus moves towards term. After birth, photoreceptors continue to mature and at the end of eight months of age, the OS of the central retina becomes larger than that of the near disc. Retina gains its full maturity at five years of age.

At about 11 weeks of gestation, the presence of NRL and NR2E3-IR nuclei is detected at the border of PCA, while IRBP-IR rods are found in scattered fashion on the border of PCA at about 13 weeks of gestation. All early rod markers are more or less visible in the mid-periphery of retina at about 14-15 weeks of gestation. At mid gestational period, peripheral retina shows more production of rod cells, as revealed by a sharp increase in ONL thickness along with the presence of ectopic photoreceptor cells. Outer plexiform layer $(\mathrm{OPL})$ is fully generated at the level of the peripheral retina at about 20 weeks of gestation. Earliest detection of ROP mRNA occurs at about 14 weeks of gestation at the margin of PCA, which occurs near the optic disc by about 20-21 weeks.

Peripherin expression is simultaneously present with ROP, while arrestin appears at about 15-16 weeks of gestation followed by TULP two weeks later.

Rod cells are typically very less in number in those who could potentially end up with RP. Foveal cones were shown to have very short outer and inner segments, while RPE cells close to the foveal cones contain numerous melanolysosomes and phagosomes, which might be due to the capability of RPE cells to phagocytose the cone outer segments. Lipofuscin granules are often found in RPE cells due to cone cells outer segment digestion $(10,11)$. Membranous whorls, which are often present in the subretinal spaces, are found not only in RP but also in other hereditary retinal degenerations (12). Whorls are probably either the product of incomplete phagocytosis by RPE cells or damaged outer segments of photoreceptor cells. However, melanolysosomes, which are present in RPE cells in the fovea and perifoveal region, can be a future predictor of autosomal dominant RP (13) or leber congenital amaurosis (14). Autophagic vacuoles in cone cells are meant for autodigestion and reflect the degradation process within the cone cells, but they can be found in normal photoreceptor cells (15) as well as following ischaemic events (16). Bundles of parallel filaments in inner segments of cones can also be found in hereditary retinal degeneration (17) or retinoblastoma (18).

Conflicts of interest: none declared.

Financial support: none declared.

Acknowledgements: EM Facility, SAIF, Department of Anatomy, All India Institute of Medical Sciences (AlIMS), New Delhi and IHC Lab, Department of Anatomy (IHC) and Department of Obstetrics and Gynaecology, AlIMS, Bhubaneswar, India.

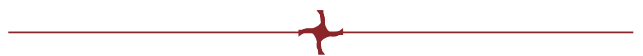

\section{R RFERENCES}

1. Curcio CA, Sloan KR, Hendrickson AE, Kalina RE. Individual variability in topography of human retinal photoreceptors.

Soc Neurosci Abstr 1986;12:636.

2. Curcio CE, Sloan KR, Kalina RE, Hendrickson A. Human photoreceptor topography. J Comp Neurol 1990;292:497-523.

3. Curcio CA, Hendrickson A. Organization and development of the primate photoreceptor mosaic.
Prog Retin Res 1991;10:90-120.

4. Provis JM, Diaz CM, Dreher B. Ontogeny of the primate fovea: a central issue in retinal development. Prog Neurobiol 1998;54:549-580.

5. Osterberg GA. Topography of the layer of rods and cones in the human retina. Acta Ophthalmologica 1935;13:1-97.

6. Hendrickson A, Kupfer C. The histogenesis of the fovea in the macaque monkey.
Investigative Ophthalmology 1976;15:746-756

7. Hendrickson AE, Yuodelis C. The morphological development of the human fovea. Ophthalmology 1984:91:603-612

8. Banerjee P, Kleyn PW, Knowles JA, et al. TULP1mutation in two extended Dominican kindreds with autosomal recessive retinitis pigmentosa. Nat Genet 1998;18:177-179. 
9. Bessant DA, Payne AM, Mitton KP, et al. A mutation in NRL is associated with autosomal dominant retinitis pigmentosa.

Nat Genet 1999;21:355-356

10. Feeney L, Grieshaber JA, Hogan MJ. Studies of human ocular pigment. In: Roben JW, editor, Eye structure, II Symposium, Stuttgart, Schattauer-Verlag, 1965, pp 535-548.

11. Hogan MJ. The retinal pigment epithelium in macular disease. Trans Am Acad Ophthalmol Otolaryngol
1972;78:64.

12. Dowling JE, Sidman RL. Inherited retinal dystrophy in the rat, J Cell Biol 1962;14:73.

13. Kolb H, Gouras P. Electron microscopic observations of human retinitis pigmentosa, dominantly inherited. Invest Ophthalmol 1974;13:487.

14. Kroll AJ, Kuwabara T. Electron microscopy of a retinal abiotrophy. Arch Ophthalmol 1964;71:683.

15. Young R. Visual cells and the concept of renewal.
Invest Ophthalmol 1976;15:700.

16. Khattab RL. Alterations in acid phosphatase bodies (lysosomes) in cat moto-neurons after asphyxiations of the spinal cord. Exp Neurol 1967;18:133.

17. Aguirre GD, Rubin LF. Pathology of hemeralopia in Alaskan malamute dog. Invest Ophthalmol 1974;13:231.

18. Popoff NA. Fliamentous alteration in photoreceptors from human eyes with retinoblastoma.

J Ultrastruct Res 1973;42:244. 